Running Head: Autonomy-Connectedness, Gender and Culture

Autonomy-Connectedness, Gender and Culture: A comparative study on the interplay of personality functioning and social context

Carla Moleiro*, Laurence Marto*, João M. Santos ${ }^{*} \&$ Inês Ratinho*

*Instituto Universitário de Lisboa ISCTE-IUL, Cis-IUL, Lisboa, Portugal

All correspondence should be sent to: Carla Moleiro, Instituto Universitário de Lisboa ISCTE-IUL, Psychology Department, Avenida das Forças Armadas (box 310), 1649-026 Lisbon - Portugal. E-mail: carla.moleiro@iscte-iul.pt; Tel: +351 217903216 


\title{
Autonomy-Connectedness, Gender and Culture: A comparative study on the interplay of personality functioning and social context
}

\begin{abstract}
Autonomy-connectedness refers to the need and ability the individual has for self-governance, as well as the capacity for intimacy and interpersonal relationships. The present study aimed to examine and compare participants $(\mathrm{N}=121)$ of four different cultural groups, namely Portuguese natives as well as Brazilian, British and Ukrainian immigrants residing in Portugal, on the different autonomy-connectedness components (Self-Awareness: SA; Sensitivity to Others: SO; Capacity for Managing New Situations: CMNS). It also aimed at investigating the relationship between autonomy-connectedness and acculturation among the groups of immigrants. The results showed significant gender differences and among the cultural groups on SO. Adherence to the heritage culture was associated with the distinct dimensions of autonomy-connectedness differently for the various groups, and time of residence was also key in how the dimensions were endorsed cross time. This study contributes to the theoretical framework of the concept of autonomy-connectedness in light of cross-cultural perspectives.
\end{abstract}

Keywords: Autonomy-Connectedness; Culture; Gender; Personality; Acculturation 


\section{I - Introduction}

Autonomy-connectedness is a recently proposed personality construct and it aims to embody a gender- and culture-sensitive notion of autonomy, rather than a value based on individualism and masculinity (Bekker, 1993; Bekker \& Van Assen, 2006). The concept was based in feminism psychoanalytic theories of gender identification (Chodorow, 1978, 1989) and attachment theory (Ainsworth \& Bowlby, 1991; Bowlby, 1969, 1973), and reflects the dialectic tension between the need and the ability that one has for self-governance, trusting oneself and be independent, as well as the necessity and the capability for intimacy, satisfactory intimate relationships and engagement in interpersonal relationships (Bekker \& van Assen, 2006; Van Assen \& Bekker, 2009).

Traditionally, the construct of autonomy has been related to the gain of independence and individualization of the self (e.g. Mahler, Pine \& Bergmann, 1975; Erikson, 1974; Kohlberg, 1984; Piaget, 1983). However, the concept is culturally diverse. Some authors (Hmel \& Pincus 2002) have highlighted that most operacionalizations of autonomy hold a social component. In fact, autonomy is developed within a social context and healthy autonomy also implicates a meaningful relationship with another, within a secure attachment (Bowlby, 1969; Bekker, 1993). Autonomy-connectedness was introduced (Bekker, 1993; Bekker \& Van Assen, 2006) as a way to acknowledge the interdependence component in understanding autonomy, going beyond the independent-dependent dichotomy. It also aimed to be proposed as a more gender- and culture-sensitive construct, not over-relying on the focus on hegemonic masculinity views of independence and detachment from others (Hmel \& Pincus, 2002). It presents a three-dimensional conceptual body - Self-awareness (SA), Sensitivity to others (SO) and Capacity for managing new situations (CMNS) - which includes both notions of independence and interdependence (Bekker, 1993). Self-awareness refers to the capacity to be aware of one's own opinions, wishes, and needs, and the ability to express these (adequately) 
in social interactions. It relates to sensitivity to others in that this second dimension reflects the sensitivity to the opinions, wishes, and needs of other people, as well as the capacity and need for intimacy. The latter dimension comprises the feeling of being at ease in new situations, flexibility, and drive for exploration not (overly) depend on familiar structures, as expected in secure attachment. Gender differences have consistently been found, with women scoring higher on the dimension of sensitivity to others (Bekker \& van Assen, 2008; Moleiro, Ratinho \& Bernardes, 2017). Cultural differences have also been identified (Bekker, ArendsTóth, \& Croon, 2011; Maas, van Assen, van Balkom, Rutten, \& Bekker, 2019; Moleiro, Ratinho \& Bernardes, 2017). Acculturation processes have been related as a good example of the resulting transformations on personality functioning (Güngör et al., 2013), in the interplay of cultural and gender values of origin and host cultures (Schwartz, Unger, Zamboaga, \& Szapocznik, 2010). Individual differences have also been explored in the work-family balance (Bekker, Willemse \& de Goeij, 2010) among both men and women, and its relation to autonomy-connectedness; in relation to the big-Five personality factors (van Assen Bekker, 2009); and as a mediator of gender differences (Bekker \& van Assen, 2017) and vulnerability for anxiety (Kunst et al., 2019), and other symptoms of psychopathology and personality (dys)functioning.

The construct of autonomy-connectedness has been mostly studied in the Dutch population, following the work of Bekker (1993). Up to this moment, to the best of our knowledge, only three studies exist which include other cultural groups. Firstly, Bekker, Arends-Tóth and Croon (2011) studied the relation between autonomy-connectedness and the adherence to cultural values of independence and interdependence, in Dutch natives, and Turkish and Moroccan immigrants residing in the Netherlands. Therefore, this study compared the levels of the three autonomy-connectedness components of immigrant respondents from countries categorized as collectivistic, with those of native Dutch 
respondents categorized as individualistic. The results showed that higher levels of original cultural preservation were positively associated with higher levels on the SO dimension and adherence to interdependent values. Furthermore, even though they had hypothesized it, the authors did not find an association between adaptation/integration to the host culture and higher levels of SA.

Secondly, Moleiro and colleagues (2016) studied a small set of populations characterized as collectivistic. They aimed to assess a Portuguese sample in terms of autonomy-connectedness, as well as differences among different cultural groups, assuming that they shared some characteristics (e.g. described as collectivistic) and differed in others (e.g. characterized in terms of values of masculinity/femininity and uncertainty avoidance; Hofstede, 1984, 2011). The study included Cape Verdean and Chinese immigrants residing in Portugal and an acculturation indicator was evaluated by time of residence in host culture (as a proxy index). The results showed differences among the cultural groups on SA, with Portuguese participants scoring higher on this dimension; gender differences were found on the SO dimension, with women consistently reporting higher scores than men; and an interaction was also significant between gender and nationality. Portuguese women scored significantly higher on SO than Portuguese men, whereas the differences between CapeVerdean men and women and between Chinese men and women residing in Portugal were more negligible - possibly due to a cultural context more characterized by gender fluid communication among these latter groups. Time of residence among the immigrant groups was found to be associated with greater scores on the SA dimension. This finding was interpreted as translating positive acculturation strategies, taking into account contextual factors (as host country participants were higher on SA) that may facilitate adaptive functioning. 
Lastly, Maas and colleagues (2019) investigated the associations between selfconstrual, acculturation, and autonomy-connectedness among over 1200 Dutch participants, $57 \%$ of which from immigrant background (Indonesia, Morocco, Turkey, South Africa, Surinam, and Dutch Antilles). The authors found that an interdependent self-construal was negatively associated with self-awareness and capacity for managing new situations; conversely, it was positively associated with sensitivity to others. These associations were true among both Dutch natives and immigrants. However, the study did not find a significant association between acculturation and autonomy-connectedness.

With the goal of further understanding the validity of the construct across other populations, and thus extend its external validity, this study intended to analyze how different groups - native Portuguese people and Brazilian, British and Ukrainian immigrants - differ in the various components of autonomy-connectedness. These groups were chosen given that they had not been included in previous investigations conducted in Portugal on autonomyconnectedness and represented reported cultural differences in Hofstede's dimensions (1980, 2011; Hofstede \& McCrae, 2004). While acknowledging criticisms to the dimension of individualism-collectivism and its (mis)uses in cross-cultural psychology as a useful explanatory mechanism for cultural differences in human behavior (see Voronov \& Singer, 2002), we utilized Hofstede's cultural dimensions as general indicators of possibly distinct cultural backgrounds. In that, we also recognized the interest in choosing cultural contexts characterized as having varying understandings of gender and of uncertainty avoidance, as these dimensions could relate to autonomy-connectedness, beyond individualism-collectivism (see Figure 1). In doing so, however, we recognize the inadequacy of a conceptualization of culture as static (Voronov \& Singer, 2002) and equivalent to group-membership (Kirmayer, 2012). 
Figure 1. Hofstede's cultural dimensions for Portugal, Ukraine, Britain and Brazil

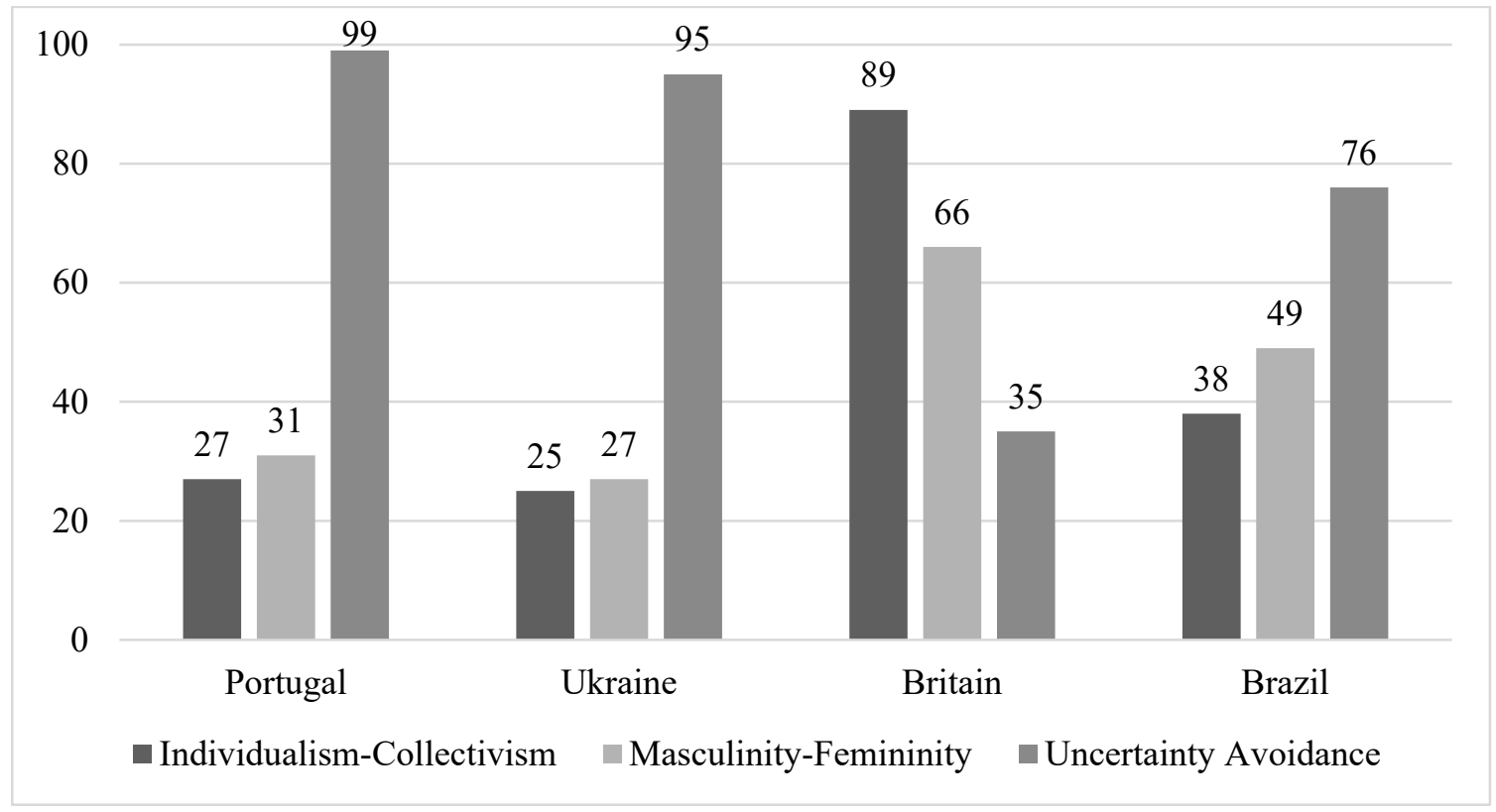

Note. Adapted from geert-hofstede.com.

We aim to explore the relationship between autonomy-connectedness and acculturation among Brazilian, British and Ukrainian immigrants residing in Portugal. Acculturation refers to the dual process of psychological and cultural change, which takes in account numerous forms of reciprocal accommodation, in consequence of the interaction among multiple cultural groups and their individual members (Safdar \& van de Vijver, 2019). At the group level, it involves changes in social organizations and in cultural practices; at the individual level, acculturation implicates changes in people's practices (Berry, 2005; Safdar \& van de Vijver, 2019). In this study, besides using length of residence as a proxy for acculturation, we were interested in understanding the relationship between acculturation and autonomy-connectedness through the degree of identification with both cultural heritage and to the host country.

By exploring the concept of autonomy-connectedness among natives and immigrants, we can provide further empirical evidence that the expectable gender differences are an etic principle of the concept of autonomy-connectedness. We can also offer an emic understanding of the construct within and between different cultures (Berry, 2005), by exploring the 
importance and influence of heritage and mainstream culture identity (Ryder, Alden \& Paulhus, 2000) and its fluidity in the interplay with the social context in varying lengths of time of residence.

In summary, with this study we hope to address two main goals, as part of the interplay between personality and interpersonal functioning, and the social and cultural context (Fiske, Kitayama, Markus, \& Nisbett, 1998; Triandis \& Suh, 2002). First, we aim to investigate how autonomy-connectedness (and its dimensions) differs among the various cultural groups in Portugal, as well as from native Portuguese participants. And second, we intend to study the relation between autonomy-connectedness and acculturation (heritage and mainstream factors), beyond time of residence. Given these goals, we can formulate the following questions:

- Can we find gender differences, with women scoring higher than men on SO, in all the groups? Are there differences among the groups, as participants' backgrounds are characterized on the masculinity vs. femininity domain?

- Are there differences in the sub-dimensions of autonomy-connectedness among the immigrant groups? Do participants from cultural backgrounds characterized as more individualistic score higher on SA and on CMNS? And lower on SO? Do the participants from groups characterized as more uncertainty avoidant display lower scores on CMNS?

- Are time of residence and acculturation strategies positively associated with higher SA scores? And how do they relate with CMNS? And SO?

\section{II - Method}

\subsection{Participants}


Sample characteristics are summarized in Table 1. This study involved the collaboration of 121 participants, of which 39 were native from Portugal and 82 were immigrants from Brazil, Britain and the Ukraine. Of the 82 immigrants encompassed by this study, 32 were Ukrainians, 21 were British and 29 were Brazilian. All participants lived in Portugal, 62 were men (51.20\%) and 59 were women (48.80\%) aged between 18 and $81(M=$ $41.40 ; S D=16.39)$. In total, $43.8 \%$ of participants were married and $38.8 \%$ were single. Regarding educational level, $28.90 \%$ of the participants had secondary education and other $28.90 \%$ had higher education, $26.40 \%$ had a post-graduate, master or doctorate degree and only $14.90 \%$ had basic education (some did not indicate their educational background).

In terms of country of origin, the distribution of participants sought to be similar across groups. The native Portuguese participants $(\mathrm{n}$ P-Group $=39)$ were aged between 20 and $68(M=33.08, S D=12.37), 67.65 \%$ were women and $32.35 \%$ were men; and $58.82 \%$ of the participants were single. From an educational point of view, $38.24 \%$ had a bachelor degree and $32.35 \%$ had a post-graduate, master or doctorate degree. Ukrainian immigrants (n UKR-group $=32)$ were aged between 21 and $61(M=38.81, S D=10.49)$. In this group, $58.82 \%$ were men and $41.18 \%$ were women. More than half of them were married $(55.88 \%)$. From an educational point of view, $44.12 \%$ had basic education and $32.35 \%$ secondary school. Of these, 55.88\% were living in Portugal for less than 10 years. Brazilian immigrants (n BRA-Group=32) were aged between 18 and $76(M=43.41, S D=16.49)$. In this group, $62.50 \%$ were men and $37.50 \%$ were women; and $46.88 \%$ were married and $37.50 \%$ were single. Half of Brazilian immigrants had the educational level of basic education. Of these, $62.5 \%$ were living in Portugal for less than 10 years, but $31.25 \%$ were living in the country for over 20 years. British immigrants (n BRI-Group =21) were aged between 31 and $81(M=$ $58.00 ; S D=16.49), 52.38 \%$ were men and $47.62 \%$ were women. In this group, $66.67 \%$ were 
married and $57.14 \%$ were postgraduates, masters or doctors. Of these, $71.43 \%$ were living in Portugal for less than 10 years.

Table 1. Characterization of the Sample of Participants per Group (Frequencies)

\begin{tabular}{|c|c|c|c|c|c|c|c|c|c|c|}
\hline & \multicolumn{2}{|c|}{ P-Group } & \multicolumn{2}{|c|}{ BRI-Group } & \multicolumn{2}{|c|}{ BRA-Group } & \multicolumn{2}{|c|}{ UKR-Group } & \multicolumn{2}{|c|}{ Total } \\
\hline $\mathrm{N}$ & & 39 & \multicolumn{2}{|c|}{21} & \multicolumn{2}{|c|}{29} & \multicolumn{2}{|c|}{32} & \multicolumn{2}{|c|}{121} \\
\hline Female & & 24 & \multicolumn{2}{|c|}{10} & \multicolumn{2}{|c|}{12} & \multicolumn{2}{|c|}{13} & \multicolumn{2}{|c|}{59} \\
\hline Male & \multicolumn{2}{|r|}{15} & \multicolumn{2}{|c|}{11} & \multicolumn{2}{|c|}{17} & \multicolumn{2}{|c|}{19} & \multicolumn{2}{|c|}{62} \\
\hline Mean Age (sd) & 33.1 & $(12.4)$ & 58.0 & $(16.5)$ & 43.4 & $(16.5)$ & 38.8 & $(10.5)$ & 41.4 & (16.4) \\
\hline \multicolumn{11}{|l|}{ Relational Status } \\
\hline Single & & 22 & & 3 & & 2 & & 10 & & \\
\hline Married/Union & & 14 & & 14 & & 4 & & 21 & & \\
\hline Divorced & & 2 & & 3 & & 1 & & 1 & & \\
\hline Widowed & & 1 & & 1 & & 2 & & 0 & & \\
\hline \multicolumn{11}{|l|}{ Education } \\
\hline None completed & & 0 & & 0 & & 1 & & 0 & & \\
\hline Elementary & & 4 & & 0 & & 3 & & 1 & & \\
\hline High School & & 9 & & 4 & & 7 & & 15 & & \\
\hline Univ/College & & 14 & & 5 & & 6 & & 10 & & \\
\hline
\end{tabular}

\subsection{Procedures and Instruments}

After an online questionnaire in Portuguese and English was developed using Qualtrics, the study was distributed online (59.5\% of participants completed the online version) and in paper versions ( $40.5 \%$ of participants completed the paper version). Dissemination of the invitation to participate was done through email, contacting relevant NGO's, which distributed either the link or the paper version of the questionnaire. Hence, participants were recruited through internet-based and snowballing sampling methods via social media platforms and 
associations. Respondents were told their participation was voluntary and anonymous, as well as the objectives set by the study. They provided informed consent and were requested to fill out the questionnaires and a socio-demographic inquiry for the characterization of the surveyed population.

2.2.1 Autonomy-Connectedness. To measure main construct, the brief AutonomyConnectedness Scale was used (ACS-30; Bekker \& Van Assen, 2006). This scale contains 30 items, divided into 3 subscales: 17 items on sensitivity towards others; 7 items on selfawareness; and 6 items on the capacity to manage new situations. Respondents had to choose their degree of agreement or disagreement on a Likert type scale from one (strongly disagree) to five (strongly agree). As aforementioned, SA refers to the awareness of one's own opinions, desires and needs as well as the ability to express them during social interactions. An example of a SA subscale item is "Usually it is easy for me to know what I like most." In Bekker and Van Assen's study (2006), this subscale showed a 0.81 Cronbach's alpha, with a satisfactory value in terms of internal consistency. SO encompasses consideration of the opinions, desires and needs of others; empathy and the ability and need for intimacy and separation. An item of this subscale is, for example, "Experiences of other people have a great impact on me." Cronbach's alpha of SO obtained by the authors was 0.83 . CMNS reflects on the sense of (dis)comfort or (in)security that people feel towards new situations and dependence on family structures. An example of this scale item is "I'm quickly at ease in new situations." The Cronbach's alpha for this subscale in the original study reveals a satisfactory internal consistency of 0.82 .

ACS-30, which was originally developed in Dutch and English, had already been adapted to the Portuguese population (Moleiro, Ratinho \& Bernardes, 2017). In this study, the Cronbach's alpha of SO and CMNS subscales showed a satisfactory internal consistency of 0.78 and 0.77 respectively. The exception was the SA subscale with an Alpha of 0.68. 
Although it is not an extraordinarily low value, it increased 0.70 by removing item 28 ("When I disagree with someone, I make it clear").

2.2.2. Acculturation. To measure the acculturation level, the Vancouver Index of Acculturation, by Ryder, Alden and Paulhus (2000) was used. This scale was formulated based on the perspective that acculturation is better understood when the weight of culture heritage and dominant culture are evaluated separately from one another and are thus able to independently suffer changes. This scale contains a total of 20 items, divided into 2 subscales: 10 items for the Heritage (cultural heritage) subscale; and 10 items for the Mainstream (the host country's culture) subscale. The items depict the various domains relevant in acculturation, including values, social relations, and adherence to traditions. An item of the Heritage subscale is, for example, "I like to participate in social events with people of my own culture." The Cronbach's alpha of the Heritage subscale ranged from 0.91 to 0.92 (Ryder, Alden \& Paulhus, 2000). An item of the Mainstream subscale is "I would you be willing to marry a native Portuguese individual". Cronbach's alpha ranged from 0.85 to 0.89 (Ryder, Alden \& Paulhus, 2000). Items are rated on a Likert type of rating scale having 9 points with responses ranging from 1 (strongly disagree) to 9 (agree).

The Vancouver Index of Acculturation, by Ryder, Alden and Paulhus (2000), to our knowledge, had not been previously adapted to the Portuguese population, making it necessary to translate it from English to Portuguese. For a final version of the scale in Portuguese, two Portuguese native speakers were needed to translate it from English to Portuguese. Then the scale was back-translated from Portuguese to English by two English native speakers, to ensure that the original meaning of the items was maintained. In our study, the Cronbach's alpha was 0.93 for the Mainstream subscale and 0.95 for the Heritage subscale. 
The answers to the questionnaire, in paper and online versions, were aggregated into a database, from which the different statistical analyses were carried out, using the Statistical Package for the Social Sciences (SPSS) - Version 23.

\section{III - Results}

The descriptive statistics on the various dimensions of autonomy-connectedness for each cultural group represented in the study are shown in Table 2.

Table 2. Means and standard deviations of Autonomy-connectedness Dimensions (SA, SO, CMNS) for each Cultural Background and Gender

\begin{tabular}{ccccccccccc}
\hline Scale & \multicolumn{2}{c}{ P-Group } & \multicolumn{2}{c}{ BRI-Group } & \multicolumn{2}{c}{ BRA-Group } & \multicolumn{2}{c}{ UKR-Group } & \multicolumn{2}{c}{ Total } \\
\cline { 2 - 11 } & Women & Men & Women & Men & Women & Men & Women & Men & Women & Men \\
& M (SD) & M (SD) & M (SD) & M (SD) & M (SD) & M (SD) & M (SD) & M (SD) & M (SD) & M (SD) \\
\hline SA & 3.29 & 3.56 & 3.62 & 3.11 & 3.75 & 3.61 & 3.42 & 3.67 & 3.47 & 3.53 \\
& $(0.80)$ & $(0.55)$ & $(0.79)$ & $(0.70)$ & $(0.51)$ & $(0.74)$ & $(0.59)$ & $(0.46)$ & $(0.71)$ & $(0.63)$ \\
SO & 3.46 & 3.11 & 2.75 & 2.85 & 3.26 & 2.83 & 3.25 & 3.11 & 3.25 & 2.97 \\
& $(0.60)$ & $(0.48)$ & $(0.43)$ & $(0.27)$ & $(0.55)$ & $(0.52)$ & $(0.44)$ & $(0.32)$ & $(0.57)$ & $(0.43)$ \\
CMNS & 3.04 & 3.29 & 3.64 & 3.15 & 3.10 & 3.50 & 3.26 & 3.19 & 3.21 & 3.30 \\
& $(0.88)$ & $(0.55)$ & $(0.82)$ & $(0.28)$ & $(0.76)$ & $(0.85)$ & $(1.25)$ & $(0.65)$ & $(0.95)$ & $(0.67)$ \\
\hline
\end{tabular}

Differences were tested in terms of gender, cultural background, and their possible interaction. In regards to the first research question, gender differences were investigated and significant differences were found for the SO subscale $(F(1,121)=5.07, p<0.05)$, with women $(M=3.25)$ obtaining in average higher values than men $(M=2.97)$, as expected. In the SA and CMNS subscales, gender differences did not reach statistical significance. Furthermore, the interaction between gender and the cultural background turned out not to be 
significant $(F(9,339)=1.26, n s)$, indicating that in all groups the scores reported by women and men revealed a similar pattern.

The second research question addressed the issue of the differences among the groups of distinct cultural backgrounds. A significant difference was found in $\operatorname{SO}(F(1,121)=4.70$, $p<0.01$ ). To explore these significant differences, Bonferroni post hoc tests were performed. Results indicated that Portuguese and Ukrainian participants scored higher than British participants on SO, and the Portuguese sub-group was also higher than the Brazilian. On the SA and CMNS subscales, the differences between the various cultural groups were not statistically significant.

Lastly, and more importantly, the third research question addressed the relation between autonomy-connectedness and acculturation and time of residence among the immigrant groups. The descriptive data on the means and standard deviations of the Length of Residence and Acculturation Index (Mainstream and Heritage) variables on the subscales of autonomy-connectedness by groups of immigrants (Brazilians, British and Ukrainian) is shown in Table 3.

Table 3. Frequencies, Means and standard deviations of Length of Residence and Acculturation Index (Mainstream and Heritage) by Immigrant Group

\begin{tabular}{ccccc}
\hline Variables & BRI-Group & BRA-Group & UKR-Group & Total \\
\hline Length of residence (freq.) & & & & \\
1 1-10 years & 15 & 20 & 19 & 55 \\
11-20 years & 4 & 2 & 11 & 18 \\
$20+$ years & 2 & 10 & 4 & 48 \\
Mainstream Acculturation Index & $5.45(1.34)$ & $6.29(1.72)$ & $6.89(1.53)$ & $6.29(1.66)$ \\
Heritage Acculturation Index & $6.56(1.74)$ & $7.09(1.09)$ & $7.28(1.56)$ & $7.04(1.46)$ \\
\hline
\end{tabular}


To investigate mean differences, 2-way ANOVAs were performed (3 cultural groups $\mathrm{x}$ 3 categories of time of residence) for each dependent variable (the subscales of autonomyconnectedness). The interaction between Immigrant Group and Length of Residence turned out to be significant in CMNS $(F(4,87)=4.38, p<0.01)$ and $\mathrm{SA}(F(4,87)=3.83, p<0.01)$ subscales.

Figure 2. Interaction between Immigrant Group and Length of Residence in the CMNS subscale

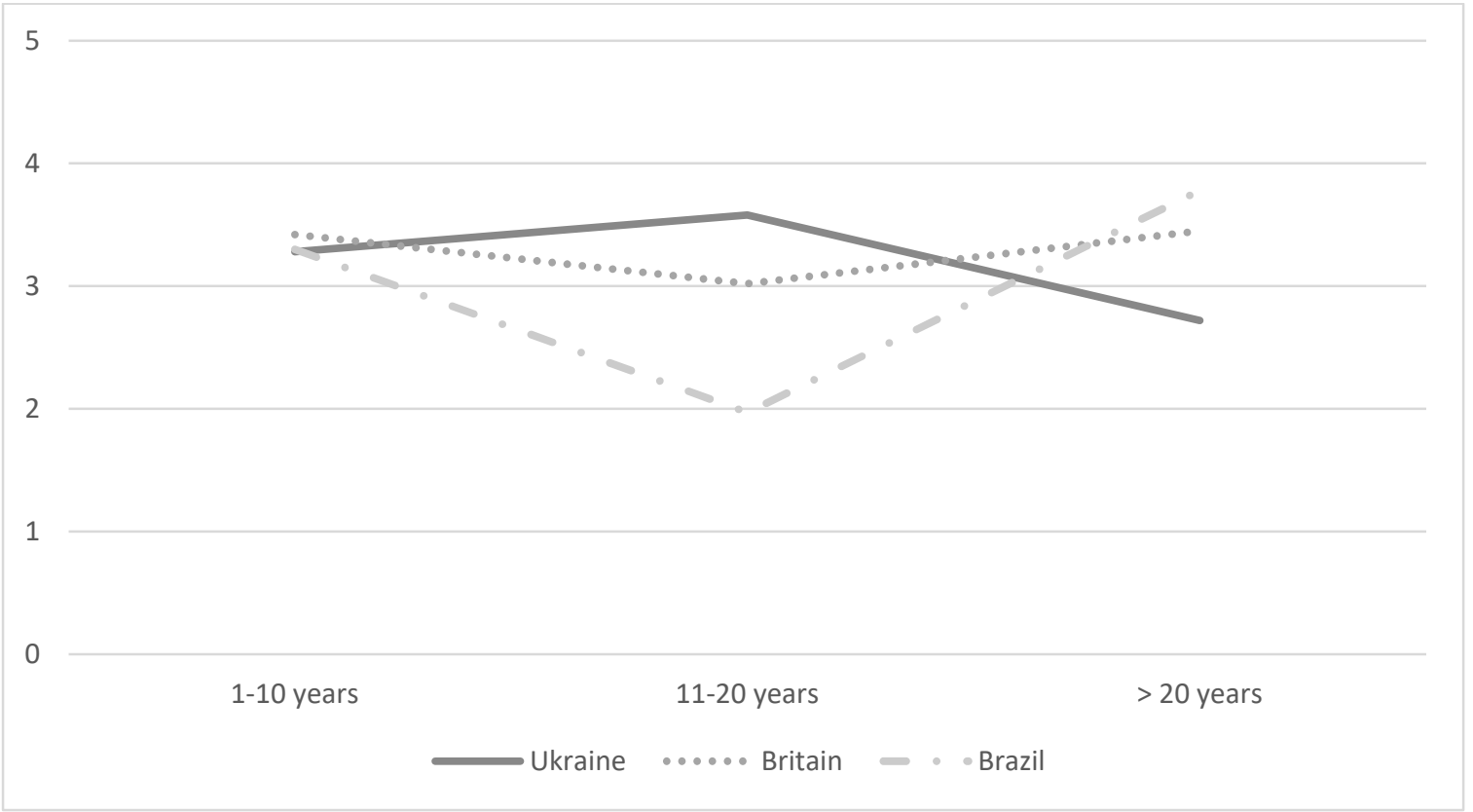

In Figure 2, it is possible to observe that, before 10 years of residence, there were no differences between cultural groups with regard to the CMNS. Nonetheless, from 11 to 20 years of length of stay, Brazilian participants revealed substantially less CMNS. From two decades onwards, the situation was reversed, and Ukrainian participants became the ones with less CMNS. Thereto, over time, the ability to manage new situations appeared to evolve differently depending on the immigrant group. In Figure 3, the situation is presented for the SA subscale, suggesting a similar pattern of findings. That is, from 11 to 20 years of 
residence, a decline among the score of Brazilian participants was observed, and from 20 years onwards, the British participants were far higher than other countries in terms of SA. Thus, time of residence seemed to play a role in personality functioning of participants, in particular, self-awareness and the capacity to manage new situations, depending on the cultural background.

Figure 3. Interaction between Immigrant Group and Length of Residence in the SA subscale

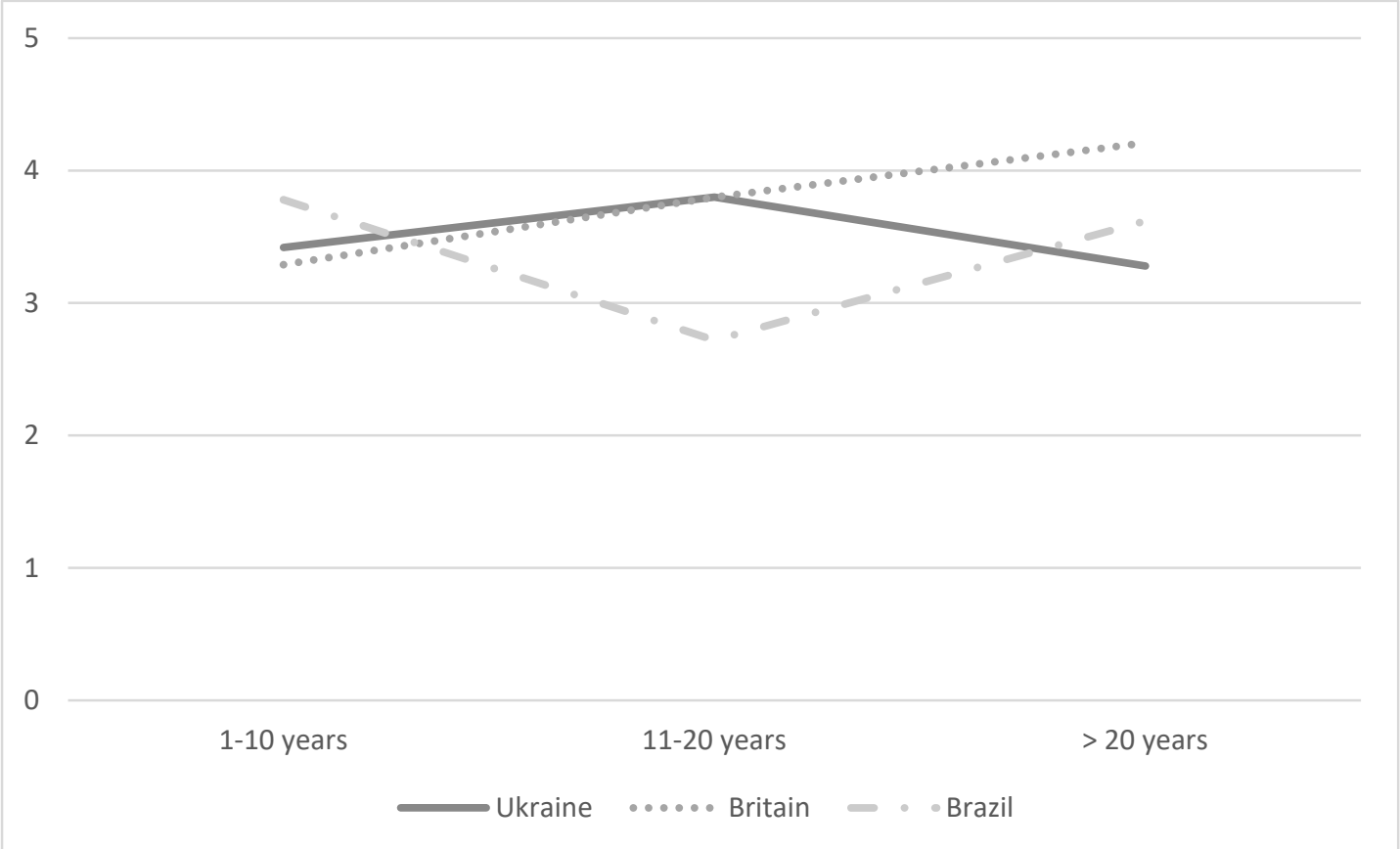

In order to perform a similar analysis with acculturation strategies, both the Mainstream Acculturation Index and the Heritage Acculturation Index were dichotomized based on the median. The effect of Heritage Acculturation Index was significant with regard to variables $\operatorname{CMNS}(F(1,88)=5.51, p<0.05)$ and SO $(F(1,88)=9.89, p<0.01)$; and the interaction between it and the Immigrant Group membership was significant for the three dependent variables: SA $(F(2,88)=4.36, p<0.05)$, $\mathrm{SO}(F(2,88)=10.64, p<0.01)$ and $\operatorname{CMNS}(F(2,88)$ $=6.56, p<0.01)$. The interaction term allowed us to better understand the effect of Heritage Acculturation on the three subscales for each country; first, it stood to our attention that for 
Brazilian participants, the greater the Heritage Acculturation Index, the greater the sensitivity towards others $(b=0.304, t(80)=3.93, p<0.01)$, and the lower the CMNS score $(b=-0.323$, $t(80)=-2.48, p<0.05)$. In the case of the British participants, as the Heritage Acculturation Index increased so did the CMNS $(b=0.472, t(80)=2.98, p<0.01)$, although the SO score was lower $(b=-0.406, t(80)=-4.31, p<0.01)$. For Ukrainians, these relationships did not reach statistical significance. In conclusion, in the case of Brazilians, the connection to the culture of origin was associated with a higher SO but to a lower CMNS. In case of the British, the connection to the culture of origin was associated with a lower SO but with higher CMNS. Results for the Mainstream Acculturation Index were not significant, that is, the relationship with the culture of the host country was not found to be associated with autonomyconnectedness dimensions.

\section{IV - Discussion}

In general, this study intended to analyze how individuals from the following groups native Portuguese people and Brazilian, British and Ukrainian immigrants - differ in the various components of autonomy-connectedness (Bekker, 1993; Bekker \& van Assen, 2006), or in other words, how people's personality functioning may be different in light of social and cultural background and current social contexts. In addition, the study aimed to explore the relationship between autonomy-connectedness and acculturation among Brazilian, British and Ukrainian immigrants, taking into consideration that cultural background is fluid and dynamic (Kirmayer, 2012), at an individual, interpersonal and group level. The main results showed that there were significant differences between the various studied cultural groups in the SO subscale, as well as gender differences. The results also showed that, for Brazilian participants, the connection to the culture of origin was associated with a higher SO, but to a lower CMNS. For the British participants, the connection to the culture of origin was 
associated with a lower SO but with higher CMNS. Consistently, the length of residence in the host country also denoted differences and over time, the CMNS and SA were found to have different patterns depending on the cultural background.

Firstly, we expected that women would obtain a higher result on autonomyconnectedness, namely, in sensitivity to others, than men. This expectation was confirmed, as significant differences were found for the SO subscale. These gender differences found in SO matched the results found in previous studies (Bekker \& Belt, 2006; Bekker \& van Assen, 2008, Bekker et al., 2011; Moleiro, Ratinho \& Bernardes, 2017) and seem to reflect a notion of autonomy which may translate developmental and gender socialization processes (Chodorow, 1978, 1989). That is, in contrast with more traditional approaches to the concept of autonomy, autonomy-connectedness embraces the interdependence dimension, which (still) has been more typically a part of women's gender socialization, more than of the hegemonic masculinity (Aboim, 2010). In our research question, we wondered if these gender differences would vary across cultural groups; in particular, how gender differences in SO would play out among cultural backgrounds described as more or less gender fluid in their roles and communication. Brazilian participants could reveal the least gender differences in SO compared with the other groups, since it has been characterized by a more intermediate score on Hofstede's cultural dimension of Masculinity vs. Femininity (Hofstede, 2011). This was not confirmed as this gender difference was found in all groups, contrarily to previously found by Moleiro et al (2017) among Cape-Verdean men and women. As more cultural groups are studied and as gender norms change for the socialization of both men and women in different cultures, further investigation on gender differences on autonomy-connectedness and its role will prove useful to understand individual and interpersonal functioning (Bekker \& van Assen, 2017). 
In regards to our second goal, we expected to find group differences based on cultural background. Specifically, given that Britain, out of all the groups, has been the one most characterized as individualistic and less collectivistic (Hofstede, 2011), British participants were expected to have a higher scores in SA and lower scores on SO. This was partially confirmed as this group showed differences only in terms of the SO subscale, with Portuguese and Ukrainian participants obtaining higher values than the British ones in this subscale. In fact, in other studies (Bekker et al., 2011; Maas at al., 2019), hypotheses based on the individualism-collectivism dimensions have not always been supported, nor the relationships between acculturation and self-awareness. These results may be relevant in the critical reviews on cultural dimensions as explanatory of cultural differences (Voronov \& Singer, 2002).

It was also expected that the length of residence and acculturation would be associated with a higher SA, but by direct effect, this was not verified; rather, we concluded that over time CMNS and SA evolve differently depending on the cultural background. In a study by Moleiro, Ratinho and Bernardes (2017), the results showed that the effect of length of residence was significant only for the SA subscale; but in the study by Bekker et al. (2011), a greater adaptation to the new culture did not contribute to an increase in self-awareness among Moroccan and Turkish migrants in the Netherlands. Our results showed that for Brazilian immigrants - whose cultural context is considered collectivist - the connection to the culture of origin is associated with increased SO and lower CMNS. In the case of the British participants, connection to the culture of origin was associated with increased CMNS but lower SO. These findings speak to the complex nature of the relationship between autonomyconnectedness and acculturation (Schwartz et al., 2010). Rather than a linear correlation, a multidimensional association might be at place, translating the diversity of psychological functioning (Fiske et al., 1998; Triandis \& Suh, 2002) - partially explaining the distinct 
results found by previous studies. The relation might not only be dependent on the measure of acculturation, but also may be different for distinct individuals and cultural groups (both migrant and host cultures), length of stay, and relation to host and heritage culture.

However, and after discussing these empirical findings, we consider it is important to recognize some limitations that may have arisen during the study. The most striking limitation evident in the study relates to the sample. Sample size (and each subgroup) was limited, due to how difficult it was to recruit immigrant participants from the targeted populations. As a consequence, the results of this study may not be representative of the different populations under study. In addition, the study of the effect of length of residence and acculturation was performed in a cross-sectional study (instead of a longitudinal design); therefore the results should be analyzed taking this into account. We also recognize the modest internal consistency obtained in this study from some of the scales, possibly also related to the small sample size; while promising, this should be a guiding result for future studies. Finally, the study reflects the immigrant populations in Portugal, which may be (and are expected to be) distinct from populations in their countries of origin. Future studies may not only have a longitudinal nature, studying autonomy-connectedness along the length of residence in the host country, but also make comparisons between populations in the countries of origin and migrants.

Still, and given the aforementioned limitations, we believe to have contributed to a better understanding of autonomy-connectedness in different cultural and social contexts. Gender differences found in SO confirmed the results of other studies (Bekker \& Belt, 2006; Bekker \& van Assen, 2008, Bekker et al., 2011; Moleiro, Ratinho \& Bernardes, 2017). A relation between autonomy-connectedness and acculturation was also found, in particular, it was found that the connection to the cultural heritage has an impact on subscales of autonomy-connectedness. 
Autonomy-Connectedness, Gender and Culture 


\section{References}

Aboim, S. (2010). Family and gender values in contemporary Europe: The attitudinal gender gap from a cross-national perspective. Portuguese Journal of Social Sciences, 9(1), 33-58.

Ainsworth, M. D. S., \& Bowlby, J. (1991). An ethological approach to personality development.American Psychologist, 46, 331-341.

Bekker, M. H. J. (1993). The development of an Autonomy scale based on recent insights into gender identity. European Journal of Personality, 7, 177-194.

Bekker, M. H. J. \& Belt, U. (2006). The Role of Autonomy-Connectedness in Depression and Anxiety. Depression and Anxiety, 23, 274-280.

Bekker, M. H. J. \& van Assen, M. (2006). A short form of the autonomy scale: Properties of the Autonomy-Connectedness Scale (ACS-30). Journal of Personality Assessment, 86, 1, 51-60.

Bekker, M. H. J. \& van Assen, M. (2008). Autonomy-Connectedness and Gender. Sex Roles, 59, $532-544$.

Bekker, M. H. J., \& van Assen, M. A. L. M. (2017). Autonomy-connectedness mediates sex differences in symptoms of psychopathology. PLoS ONE, 12(8), [e0181626].

Bekker, M. H. J., Arends-Tóth, J. V.\& Croon, M. A. (2011). Autonomy-connectedness, acculturation, and independence-interdependence among various cultural groups in a multicultural society. International Journal of Intercultural Relations. 35(3), 368-376.

Bekker, M. H. J., Willemse, J. J. P., \& de Goeij, J. W. J. M. (2010). The role of individual differences in particular autonomy-connectedness in women's and men's work-family balance. Women \& Health, 50(3), 241-261.

Berry, J. W. (2005). Acculturation: Living successfully in two Cultures. International Journal of Intercultural Relations, 29, 697-712.

Bowlby, J. (1969). Attachment and Loss: Volume I: Attachment. New York: Basic Books. 
Bowlby, J. (1973). Attachment and Loss: Volume II: Separation. New York: Basic Books.

Chodorow, N. (1978). The Reproduction of Mothering.Berkeley: University of California Press.

Chodorow, N. (1989). Feminism and Psychoanalytic Theory. Cambridge: Polity Press.

Erikson, E.H. (1974). Dimensions of a New Identity. New York: Norton.

Fiske, A. P., Kitayama, S., Markus, H.R., \& Nisbett, R.E. (1998). The cultural matrix of social psychology. In D. Gilbert, S. Fiske, \& G. Lindzey (Eds.). The handbook of social psychology. (pp. 915-981). San Francisco: McGraw-Hill.

Güngör, D., Bornstein, M. H., De Leersnyder, J., Cote, L., Ceulemans, E., \& Mesquita, B. (2013). Acculturation of Personality: A Three-Culture Study of Japanese, Japanese Americans, and European Americans. Journal of Cross-Cultural Psychology, 44(5), 701-718.

Hmel, B. A., \& Pincus, A. L. (2002). The meaning of autonomy: On and beyond the interpersonal circumplex. Journal of Personality 70: 277-310.

Hofstede, G. \& McCrae, R. R. (2004). Culture and personality revisited: Linking traits and dimensions of culture. Cross-Cultural Research, 38, 52-88.

Hofstede, G. (2011). Dimensionalizing Cultures: The Hofstede Model in Context. Online Readings in Psychology and Culture, 2(1) http://dx.doi.org/10.9707/2307-0919.1014.

Hofstede, G. H. (1980). Culture's consequences: International differences in work related values. Beverly Hills, CA: Sage.

Kirmayer, LJ. (2012). Rethinking cultural competence. Transcultural Psychiatry, 49, 149-64.

Kohlberg, L. (1984). Essays in moral development: Vol. II. The psychology of moral development. New York: Harper \& Row.

Kunst, L. E., Maas, J., van Assen, M. A. L. M., Van der Heijden, W., \& Bekker, M. H. J. (2019). Autonomy deficits as vulnerability for anxiety: Evidence from two laboratory-based studies. Anxiety, Stress and Coping, 32(3), 244-258. 
Mahler, M. A., Pine, F., \& Bergman, A. (1975). The psychological birth of the human infant: Symbiosis and individuation. New York: Basic Books.

Maas, J., van Assen, M.A.L.M., van Balkom, A.J.L.M., Rutten, E.A.P. \& Bekker, M. (2019). Autonomy-Connectedness, Self-Construal, and Acculturation: Associations with Mental Health in a Multicultural Society. Journal of Cross-Cultural Psychology, 50(1), 80-99.

Moleiro, C., Ratinho, I., \& Benardes, S. (2017). Autonomy-Connectedness in Collectivistic Cultures: An exploratory cross-cultural study among Portuguese natives, Cape-Verdean and Chinese people residing in Portugal. Personality and Individual Differences, 104, 23-28.

Piaget, J. (1983). Piaget's theory. In P. (Ed.), Handbook of child psychology, Vol. 1. (pp. 103128). New York: Wiley.

Ryder, A. G., Alden, L. E., \& Paulhus, D. L. (2000). Is acculturation unidimensional or Bidimensional? A head-to-head comparison in the prediction of personality, self-identity, and adjustment. Journal of Personality and Social Psychology, 79(1), 49-65.

Safdar, S. \& van de Vijver, F.J.R. (2019). Acculturation and its application: A conceptual review and analysis. In K.C. O’Doherty and D. Hodgetts (Eds), The SAGE Handbook of Applied Social Psychology (pp. 3-22). London: SAGE.

Schwartz, S. J., Unger, J. B., Zamboanga, B. L., \& Szapocznik, J. (2010). Rethinking the Concept of Acculturation: Implications for Theory and Research.The American Psychologist, 65(4), 237-251.

Triandis, H. C., \& Suh, E. M. (2002). Cultural influences on personality. Annual Review of Psychology, 53, 133-160.

Van Assen, M. A. L. M., \& Bekker, M. H. J. (2009). Sex differences in autonomyconnectedness: The role of personality factors. Personality and Individual Differences, 47, $12-17$. 
Voronov, M. \& Singer, J.A. (2002). The myth of Individualism-Collectivism: A critical review. The Journal of Social Psychology, 142(4), 461-480. 